\title{
A REFINEMENT OF AN INEQUALITY OF THE BROTHERS MARKOFF
}

BY

\author{
R. J. DUFFIN AND A. C. SCHAEFFER
}

\section{INTRODUCTION}

Suppose that $f(x)$ is a polynomial of degree $n$ such that $|f(x)| \leqq 1$ in the interval $(-1,1)$. Then according to a theorem $\left(^{1}\right)$ of A. Markoff $\left|f^{\prime}(x)\right| \leqq n^{2}$ in the same interval. This bound is actually attained by the Tchebychef polynomial $T_{n}(x)=\cos \left(n \cos ^{-1} x\right)$. A later theorem $\left(^{2}\right)$ of $\mathrm{W}$. Markoff gives the best possible bounds for the higher derivatives of $f(x)$

$$
\left|f^{(p)}(x)\right| \leqq \frac{n^{2}\left(n^{2}-1^{2}\right) \cdots\left(n^{2}-(p-1)^{2}\right)}{1 \cdot 3 \cdot 5 \cdots(2 p-1)}, \quad-1 \leqq x \leqq 1 .
$$

We show in this paper that the condition $|f(x)| \leqq 1$ in the whole interval is unnecessarily restrictive. Instead it is only necessary to assume that $|f(x)| \leqq 1$ at the $n+1$ points $x=\cos (k \pi / n) ; k=0,1,2, \cdots, n$, and the conclusions of Markoffs' theorem are unchanged. These are exactly the points at which $T_{n}(x)$ attains its maximum in the interval $(-1,1)$.

In a previous paper $\left({ }^{3}\right)$ we showed that under the assumptions of Markoffs' theorem all derivatives of $f(x)$ must satisfy

$$
\left|\frac{d^{p}}{d x^{p}} f(x)\right| \leqq\left|\frac{d^{p}}{d x^{p}} e^{i n \arccos x}\right|, \quad-1 \leqq x \leqq 1 .
$$

For $p=1$ this reduces to an inequality of S. Bernstein

$$
\left|f^{\prime}(x)\right| \leqq n\left(1-x^{2}\right)^{-1 / 2}
$$

Using (1) it is possible to deduce W. Markoff's result. However the proof which is given here is simpler. Actually (1) is not true under the weaker conditions which are assumed in the present paper. The W. Markoff inequalities are obtained here as a consequence of a more general inequality concerning Lagrange interpolation.

Presented to the Society, September 8, 1939, received by the editors September 10, 1940.

(1) A. Markoff, Sur une question posée par Mendeleieff, Bulletin of the Academy of Sciences of St. Petersburg, vol. 62 (1889), pp. 1-24.

(2) W. Markoff, Über Polynome, die in einen gegebenen Intervalle möglichst wenig von Null abweichen, Mathematische Annalen, vol. 77 (1916), pp. 213-258. The original appeared in Russian in 1892.

(3) R. J. Duffin and A. C. Schaeffer, On some inequalities of S. Bernstein and W. Markoff for derivatives of polynomials, Bulletin of The American Mathematical Society, vol. 44 (1938), pp. 289-297. 


\section{AN INEQUAlity of INTERPolation}

Suppose $g(z)$ is a polynomial of degree $n$ with $n$ distinct real zeros lying in the interval $(a, b)$ of the real axis,

$$
\begin{aligned}
g(z) & =c \prod_{\nu=1}^{n}\left(z-\lambda_{\nu}\right), & c \neq 0 ; \\
g^{\prime}(z)=\sum_{\nu=1}^{n} \frac{g(z)}{z-\lambda_{\nu}} & &
\end{aligned}
$$

We seek some way of determining the maximum of the non-analytic function

$$
M(x)=\sum_{\nu=1}^{n}\left|\frac{g(x)}{x-\lambda_{\nu}}\right|
$$

when $x$ lies in the interval $(a, b)$. By making a certain hypothesis concerning the behavior of $g(z)$ in the strip $a \leqq \Re(z) \leqq b$ (inequality (4) of Theorem I) we obtain the elegant solution

$$
M(x) \leqq M(b)=\left|g^{\prime}(b)\right|, \quad a \leqq x \leqq b .
$$

This result is equivalent to the following statement: suppose that $f(z)$ is any polynomial of degree $n$ whose derivative satisfies the condition $\left|f^{\prime}(x)\right|$ $\leqq\left|g^{\prime}(x)\right|$ at the zeros of $g(x)$; then (with the same restrictions as before regarding $g(z)$ )

$$
\left|f^{\prime}(x)\right| \leqq\left|g^{\prime}(b)\right|, \quad a \leqq x \leqq b .
$$

The equivalence is an immediate consequence of the Lagrange interpolation formula; we have

$$
f^{\prime}(z)=\sum_{\nu=1}^{n} \frac{f^{\prime}\left(\lambda_{\nu}\right)}{g^{\prime}\left(\lambda_{\nu}\right)} \frac{g(z)}{z-\lambda_{\nu}} .
$$

Hence, under the conditions of the last statement, if $x$ is any point in $(a, b)$

$$
\max \left|f^{\prime}(x)\right|=\sum_{\nu=1}^{n}\left|\frac{g(x)}{x-\lambda_{\nu}}\right| .
$$

We shall also be concerned with finding bounds for the higher derivatives of the polynomial $f(z)$, and to accomplish this we need the following lemma.

LEMмa I. If $g(z)=c \prod_{\nu=1}^{n}\left(z-\lambda_{\nu}\right), c \neq 0$, is a polynomial of degree $n$ with $n$ distinct real zeros and if $f(z)$ is a polynomial of degree $n$ such that

$$
\left|f^{\prime}\left(\lambda_{\nu}\right)\right| \leqq\left|g^{\prime}\left(\lambda_{\nu}\right)\right| ; \quad \nu=1,2,3, \cdots, n,
$$

then for $p=1,2,3, \cdots, n$ we must have at the zeros of $g^{(p-1)}(z)$

$$
\left|f^{(p)}(z)\right| \leqq\left|g^{(p)}(z)\right| \text {. }
$$


Proof. For $p=1$ inequality (2) is simply a restatement of the conditions of the lemma; so consider the case $p=2$. The Lagrange interpolation formula gives

$$
\frac{f^{\prime}(z)}{g(z)}=\sum_{\nu=1}^{n} \frac{f^{\prime}\left(\lambda_{\nu}\right)}{g^{\prime}\left(\lambda_{\nu}\right)} \frac{1}{z-\lambda_{\nu}}=\sum_{\nu=1}^{n} \frac{\delta_{\nu}}{z-\lambda_{\nu}}
$$

where by the hypotheses of the lemma, $\left|\delta_{\nu}\right| \leqq 1$. There is a similar expression for $g^{\prime}(z)$ in which each $\delta_{\nu}$ is equal to 1 . Differentiating (3) we obtain

$$
\left\{f^{\prime \prime}(z) g(z)-f^{\prime}(z) g^{\prime}(z)\right\} /\{g(z)\}^{2}=-\sum_{\nu=1}^{n} \frac{\delta_{\nu}}{\left(z-\lambda_{v}\right)^{2}} .
$$

Thus at the points where $g^{\prime}(z)=0$ we have

$$
\left|\frac{f^{\prime \prime}(z)}{g(z)}\right|=\left|\sum_{\nu=1}^{n} \frac{\delta_{\nu}}{\left(z-\lambda_{\nu}\right)^{2}}\right| \leqq \sum_{\nu=1}^{n} \frac{1}{\left(z-\lambda_{\nu}\right)^{2}}=\left|\frac{g^{\prime \prime}(z)}{g(z)}\right|
$$

and it follows that Lemma I is true for $p=2$.

The proof for $p>2$ is by induction. Let $\left|f^{(p)}(x)\right| \leqq\left|g^{(p)}(x)\right|$ at the zeros of $g^{p-1}(x)$ (they are real and distinct). By applying the previous argument to $f^{(p)}(x)$ and $g^{(p)}(x)$ instead of $f^{\prime}(x)$ and $g^{\prime}(x)$ we obtain

$$
\left|f^{(p+1)}(x)\right| \leqq\left|g^{(p+1)}(x)\right|
$$

at the zeros of $g^{(p)}(x)$. This completes the induction.

TheOREM I. Let $g(z)$ be a polynomial of degree $n$ with $n$ distinct real zeros to the left of the point $b$ on the real axis, and suppose that in a strip of the complex. plane it satisfies the inequality

$$
|g(x+i y)| \leqq|g(b+i y)| ; \quad \cdot \quad a \leqq x \leqq b,-\infty<y<\infty .
$$

Then if $f(z)$ is a polynomial of degree $n$ with real coefficients such that

$$
\left|f^{\prime}(x)\right| \leqq\left|g^{\prime}(x)\right| \text { wherever } g(x)=0
$$

the derivatives of $f(z)$ and $g(z)$ must satisfy

$$
\begin{aligned}
& \left|f^{(p)}(x+i y)\right| \leqq\left|g^{(p)}(b+i y)\right| ; \\
& \quad a \leqq x \leqq b,-\infty<y<\infty, p=1,2,3, \cdots, n .
\end{aligned}
$$

Proof. To prove Theorem I we shall first show that at a fixed point $x_{1}+i y_{1}$ in the strip

$$
\left|f^{\prime}\left(x_{1}+i y_{1}\right)\right| \leqq\left|g^{\prime}\left(b+i y_{1}\right)\right| .
$$

Let $g(z)=c \prod_{\nu=1}^{\infty}\left(z-\lambda_{\nu}\right), c \neq 0$, where without loss of generality we suppose that $c$ is real. 
Let $h(z)$ be a new polynomial with the same leading coefficient as $g(z)$, and whose roots are obtained by reflecting about $x_{1}$ those roots of $g(z)$ which lie to the right of $x_{1}$. Thus

where

$$
h(z)=c \prod_{\nu=1}^{n}\left(z-\beta_{v}\right)
$$

$$
\beta_{\nu}=\left\{\begin{array}{l}
2 x_{1}-\lambda_{\nu} \text { if } \lambda_{\nu}>x_{1}, \\
\lambda_{\nu} \text { if } \lambda_{\nu} \leqq x_{1} .
\end{array}\right.
$$

Then on the line $z=x_{1}+i y,-\infty<y<\infty$, we must have $\left|z-\beta_{\nu}\right|=\left|z-\lambda_{\nu}\right|$ so

$$
\left|h\left(x_{1}+i y\right)\right|=\left|g\left(x_{1}+i y\right)\right| \text {. }
$$

We now show that at the point $x_{1}+i y_{1}$ the derivative of $h(z)$ is at least as large as the derivative of $f(z)$. Whether or not the roots of $h(z)$ are distinct we may write

$$
\frac{h^{\prime}(z)}{h(z)}=\sum_{\nu=1}^{n} \frac{1}{z-\beta_{\nu}}
$$

and $f^{\prime}(z)$ is given by the interpolation formula (3), where now the numbers $\delta_{\nu}$ satisfy $-1 \leqq \delta_{\nu} \leqq 1$. Comparing the right-hand sides of (3) and (9) we have

$$
\begin{aligned}
\left|\sum_{\nu=1}^{n} \frac{\delta_{\nu}}{x_{1}+i y_{1}-\lambda_{\nu}}\right| & =\left|\sum \frac{\delta_{\nu}\left(x_{1}-\lambda_{\nu}\right)}{\left(x_{1}-\lambda_{\nu}\right)^{2}+y_{1}{ }^{2}}-i \sum \frac{y_{1} \delta_{\nu}}{\left(x_{1}-\lambda_{\nu}\right)^{2}+y_{1}{ }^{2}}\right| \\
& \leqq\left|\sum \frac{x_{1}-\beta_{\nu}}{\left(x_{1}-\beta_{\nu}\right)^{2}+y_{1}{ }^{2}}-i \sum \frac{y_{1}}{\left(x_{1}-\beta_{\nu}\right)^{2}+y_{1}{ }^{2}}\right| \\
& =\left|\sum \frac{1}{x_{1}-\beta_{\nu}+i y_{1}}\right|
\end{aligned}
$$

since by construction $\left|x_{1}-\lambda_{\nu}\right|=x_{1}-\beta_{\nu}$. Since $g(z)$ and $h(z)$ have the same absolute magnitude at $x_{1}+i y_{1}$ it follows that $\left|h^{\prime}\left(x_{1}+i y_{1}\right)\right| \geqq\left|f^{\prime}\left(x_{1}+i y_{1}\right)\right|$.

Let $\alpha,|\alpha|<1$, be any complex constant and let

$$
\phi(z)=g(z)-\alpha h\left(z+x_{1}-b\right) .
$$

Let $\Gamma$ be the simple closed curve consisting of a segment of the line $z=b+i y$ and the portion of a circle with center at $b$ and radius $\rho$ which lies to the right of this line. Relations (4) and (8) show that on the straight line segment of $\Gamma$

$$
|g(z)|>\left|\alpha h\left(z+x_{1}-b\right)\right| \text {. }
$$

If $\rho$ is sufficiently large the same inequality is true for the circular portion of $\Gamma$ since $g(z)$ and $h(z)$ have the same leading coefficient. Thus Rouché's theorem states that $\phi(z)$ and $g(z)$ have the same number of zeros inside $\Gamma$. We conclude that $\phi(z)$ has no zeros on or to the right of the line $b+i y$. The 
last statement, according to a theorem of Gauss, implies that $\phi^{\prime}(z)$ has no zeros on the line $b+i y$. Thus for $|\alpha|<1$

$$
g^{\prime}\left(b+i y_{1}\right)-\alpha h^{\prime}\left(x_{1}+i y_{1}\right) \neq 0 .
$$

It follows immediately that

$$
\left|g^{\prime}\left(b+i y_{1}\right)\right| \geqq\left|h^{\prime}\left(x_{1}+i y_{1}\right)\right| \geqq\left|f^{\prime}\left(x_{1}+i y_{1}\right)\right| .
$$

This proves the theorem for $p=1$. We turn now to the case $p>1$.

Applying Theorem I for $p=1$ when $f(z)$ is the function $g(z)$ itself inequality (6) shows that

$$
\left|g^{\prime}(x+i y)\right| \leqq\left|g^{\prime}(b+i y)\right| ; \quad a \leqq x \leqq b,-\infty<y<\infty .
$$

Thus $g^{\prime}(z)$ satisfies all the requirements which in Theorem I are imposed on the interpolating polynomial $g(z)$ (with $n$ replaced by $n-1$ ), and by Lemma I, $\left|f^{\prime \prime}(x)\right| \leqq\left|g^{\prime \prime}(x)\right|$ at the $n-1$ zeros of $g^{\prime}(x)$. Applying Theorem I to $f^{\prime}(x)$ instead of $f(x)$ in the case $p=1$, for which it has already been proved true, we have

$$
\left|f^{\prime \prime}(x+i y)\right| \leqq\left|g^{\prime \prime}(b+i y)\right| ; \quad a \leqq x \leqq b,-\infty<y<\infty ;
$$

which proves that inequality (6) is true for $p=2$. Repetition of this argument completes the proof for larger values of $p$.

Remark to Theorem I. If $f(z)$ and $g(z)$ satisfy the same conditions as in Theorem I except the condition that $f(x)$ has real coefficients, then for $p=1,2,3, \cdots, n$

$$
\left|f^{(p)}(x)\right| \leqq\left|g^{(p)}(b)\right|, \quad a \leqq x \leqq b .
$$

Proof. After differentiating (3) $p-1$ times we obtain

$$
f^{(p)}(x)=\sum_{\nu=1}^{n} \delta_{\nu} \frac{d^{p-1}}{d x^{p-1}}\left(\frac{g(x)}{x-\lambda_{\nu}}\right)
$$

where $\left|\delta_{\nu}\right|=\left|f^{\prime}\left(\lambda_{\nu}\right) / g^{\prime}\left(\lambda_{\nu}\right)\right| \leqq 1$. It is evident that if $x$ is any point in the interval $(a, b), f^{(p)}(x)$ attains its maximum when $\delta_{\nu}= \pm 1$. The Remark then follows from Theorem I.

\section{A PROPERTy OF TCHEBYCheF POLyNomials}

The Tchebychef polynomials $T_{n}(x)$ which are defined by

$$
T_{n}(x)=\cos n \theta, \quad x=\cos \theta,
$$

are polynomials in $x$ of degree $n$ and have $n$ real distinct roots in the interval $(-1,1)$. They may be written

$$
T_{n}(z)=c \prod_{\nu=1}^{n}\left(z-\cos \theta_{\nu}\right)
$$


where $\theta_{\nu}=\left(\nu-\frac{1}{2}\right) \pi / n ; \nu=1,2, \cdots, n$ and $c=2^{n-1}$, and they satisfy the differential equation

$$
\left(1-z^{2}\right) T_{n}^{\prime \prime}(z)-z T_{n}^{\prime}(z)+n^{2} T_{n}(z)=0 .
$$

It will be shown that $T_{n}(z)$ satisfies the conditions which in Theorem $\mathrm{I}$ are imposed on the interpolating polynomial $g(z)$ where we take $a=-1, b=1$. The proof depends on the following lemma.

LEMMA II. Let $\left\{\alpha_{i}\right\}$ be a sequence of $2 n$ non-negative numbers and let $\left\{\alpha_{i}^{\prime}\right\}$ be a rearrangement of this sequence according to magnitude,

$$
\alpha_{1}^{\prime} \geqq \alpha_{2}^{\prime} \geqq \alpha_{3}^{\prime} \geqq \cdots \geqq \alpha_{2 n}^{\prime} \geqq 0 .
$$

Then if $t \geqq 0$

$$
\left(\alpha_{1} \alpha_{2}+t\right)\left(\alpha_{3} \alpha_{4}+t\right) \cdots\left(\alpha_{2 n-1} \alpha_{2 n}+t\right)
$$

is not greater than

$$
\left(\alpha_{1}^{\prime} \alpha_{2}^{\prime}+t\right)\left(\alpha_{3}^{\prime} \alpha_{4}^{\prime}+t\right) \cdots\left(\alpha_{2 n-1}^{\prime} \alpha_{2 n}^{\prime}+t\right) .
$$

Proof. If $\alpha_{1}$ and $\alpha_{3}$ are as large as any of the $\alpha$ 's then

$$
\left(\alpha_{1} \alpha_{3}+t\right)\left(\alpha_{2} \alpha_{4}+t\right)-\left(\alpha_{1} \alpha_{2}+t\right)\left(\alpha_{3} \alpha_{4}+t\right)=t\left(\alpha_{1}-\alpha_{4}\right)\left(\alpha_{3}-\alpha_{2}\right) \geqq 0 .
$$

This shows that the $\alpha$ 's in (12) can be rearranged so that the two largest occur in the same factor without decreasing (12). Then the two largest of the remaining $\alpha$ 's may be brought into the same factor without decreasing (12). And so on.

Lemмa III. The Tchebychef polynomials satisfy the inequality

$$
\left|T_{n}(x+i y)\right| \leqq\left|T_{n}(1+i y)\right| ; \quad-1 \leqq x \leqq 1,-\infty<y<\infty .
$$

Proof. First,

$$
\left|T_{n}(x+i y)\right|^{2}=c^{2} \prod_{\nu=1}^{n}\left\{\left(x-\cos \theta_{\nu}\right)^{2}+y^{2}\right\} .
$$

If $-1 \leqq x \leqq 1$ we may write $x=\cos \theta, \theta$ real. Then we obtain

$$
\left|T_{n}(x+i y)\right|^{2}=c^{2} \prod_{\nu=1}^{n}\left\{\frac{1}{4}\left|e^{i \theta}-e^{-i \theta_{\nu}}\right|^{2}\left|e^{i \theta}-e^{i \theta_{\nu}}\right|^{2}+y^{2}\right\} .
$$

Geometrically, $e^{ \pm i \theta_{\nu}}, \nu=1,2,3, \cdots, n$, represent $2 n$ points equally distributed about the unit circle. Connect these points by chords to the point $e^{i \theta}$. Then the lengths of these $2 n$ chords are given by $\left|e^{i \theta}-e^{ \pm i \theta_{\nu}}\right|$. If $\theta$ is increased or decreased by any multiple of $\pi / n$ we obtain a new set of chords but the aggregate of their lengths is unchanged. 
Choose $\phi$ such that

$$
\phi \equiv \theta(\bmod \pi / n), \quad-\pi /(2 n) \leqq \phi \leqq \pi /(2 n) .
$$

If $x^{*}=\cos \phi$ then

$$
T_{n}\left(x^{*}+i y\right)=c^{2} \prod_{\nu=1}^{n}\left\{\frac{1}{4}\left|e^{i \phi}-e^{-i \theta_{\nu}}\right|^{2}\left|e^{i \phi}-e^{i \theta_{\nu}}\right|^{2}+y^{2}\right\}
$$

where the numbers $\left|e^{i \phi}-e^{ \pm i \theta_{\nu}}\right|^{2}$ are simply a rearrangement of the numbers $\left|e^{i \theta}-e^{ \pm i \theta_{\nu}}\right|^{2}$ appearing in (13).

We observe that Lemma II is directly applicable to the products (14) and (13). Since, as seen geometrically, the pairing of the numbers $\left|e^{i \phi}-e^{ \pm i \theta_{\nu}}\right|^{2}$ in (14) is by magnitude, we have

$$
\left|T_{n}(x+i y)\right|^{2} \leqq\left|T_{n}\left(x^{*}+i y\right)\right|^{2} .
$$

By construction $x^{*}+i y$ lies in the strip $\cos \theta_{1} \leqq x \leqq 1$ where $\cos \theta_{1}$ is the right most zero of $T_{n}(x)$. Thus the distance from any roots to $x^{*}+i y$ is no greater than the distance from the same root to $1+i y$, so (10) gives

$$
\left|T_{n}\left(x^{*}+i y\right)\right| \leqq\left|T_{n}(1+i y)\right| \text {. }
$$

This completes the proof of Lemma III. that

Pólya and Szegö prove $\left({ }^{4}\right)$ that if $P(x)$ is a polynomial of degree $n-1$ such

$$
\left(1-x^{2}\right)^{1 / 2}|P(x)| \leqq 1, \quad-1 \leqq x \leqq 1,
$$

then

$$
|P(x)| \leqq n, \quad-1 \leqq x \leqq 1 .
$$

We show that if (15) is satisfied at the $n$ points $x=\cos \left(\pi\left(\nu-\frac{1}{2}\right) / n\right)$; $\nu=1,2, \ldots, n$ then (16) is still true. We use Theorem I with $g(x)=T_{n}(x)$. When $T_{n}(x)=0, T_{n}^{\prime}(x)=n\left(1-x^{2}\right)^{-1 / 2}$ so we may write $f^{\prime}(x)=n P(x)$. Thus for $-1 \leqq x \leqq 1$,

$$
|n P(x)| \leqq T_{n}^{\prime}(1)=n^{2} .
$$

\section{MARKoffs' INEQUALITIES}

Lemma IV. Let $f(x)$ be a polynomial of degree $n$ satisfying $|f(x)| \leqq 1$ at $x=\cos (\nu \pi / n) ; \nu=0,1,2,3, \cdots, n$. Then

$$
\left|f^{\prime}(x)\right| \leqq n\left(1-x^{2}\right)^{-1 / 2} \quad \text { at } \quad x=\cos \left(\pi\left(\nu-\frac{1}{2}\right) / n\right) ; \quad \nu=1,2,3, \cdots, n .
$$

The equality can occur only if $f(x) \equiv \gamma T_{n}(x)$ for some constant $\gamma,|\gamma|=1$. 80.

(4) G. Pólya and G. Szegö, Aufgaben und Lehrsätze aus der Analysis, vol. 2, p. 90, Problem 
Proof. Let

$$
\psi(x)=\left(1-x^{2}\right) T_{n}^{\prime}(x)=a \prod_{\nu=0}^{n}(x-\cos (\nu \pi / n)) .
$$

Then by (11) $\psi^{\prime}(x)=-x T_{n}^{\prime}(x)-n^{2} T_{n}(x)$. Differentiating the Lagrange interpolation formula for $f(x)$ gives, if $\lambda_{\nu}=\cos (\nu \pi / n)$,

$$
f^{\prime}(x)=\sum_{\nu=0}^{n} \frac{f\left(\lambda_{\nu}\right)}{\psi^{\prime}\left(\lambda_{\nu}\right)} \frac{\left(x-\lambda_{\nu}\right) \psi^{\prime}(x)-\psi(x)}{\left(x-\lambda_{\nu}\right)^{2}} .
$$

At the zeros of $T_{n}(x)$ this reduces to

$$
f^{\prime}(x)=-T_{n}^{\prime}(x) \sum_{0}^{n} \frac{f\left(\lambda_{\nu}\right)}{\psi^{\prime}\left(\lambda_{\nu}\right)} \frac{1-x \lambda_{\nu}}{\left(x-\lambda_{\nu}\right)^{2}}
$$

since at these points

$$
\begin{aligned}
\left(x-\lambda_{\nu}\right) \psi^{\prime}(x)-\psi(x) & =-x\left(x-\lambda_{\nu}\right) T_{n}^{\prime}(x)-\left(1-x^{2}\right) T_{n}^{\prime}(x) \\
& =-\left(1-x \lambda_{\nu}\right) T_{n}^{\prime}(x) .
\end{aligned}
$$

In the same way, we obtain at the zeros of $T_{n}(x)$,

$$
T_{n}^{\prime}(x)=-T_{n}^{\prime}(x) \sum_{0}^{n} \frac{T_{n}\left(\lambda_{\nu}\right)}{\psi^{\prime}\left(\lambda_{\nu}\right)} \frac{1-x \lambda_{\nu}}{\left(x-\lambda_{\nu}\right)^{2}}
$$

and since $T_{n}\left(\lambda_{\nu}\right)$ and $\psi^{\prime}\left(\lambda_{\nu}\right)$ are of opposite sign this gives

$$
T_{n}^{\prime}(x)=T_{n}^{\prime}(x) \sum_{0}^{n}\left|\frac{1}{\psi^{\prime}\left(\lambda_{\nu}\right)}\right| \frac{1-x \lambda_{\nu}}{\left(x-\lambda_{\nu}\right)^{2}} .
$$

Now $\left|f\left(\lambda_{v}\right)\right| \leqq 1$, so comparing (17) and (18) we obtain

$$
\left|f^{\prime}(x)\right| \leqq\left|T_{n}^{\prime}(x)\right| \text { wherever } T_{n}(x)=0 .
$$

The equality occurs only if

$$
f\left(\lambda_{\nu}\right)=\gamma T_{n}\left(\lambda_{v}\right) ; \quad \nu=0,1,2, \cdots, n,
$$

where $\gamma$ is a constant, $|\gamma|=1$. This proves the lemma.

THEOREM II. If $f(z)$ is a polynomial of degree $n$ with real coefficients and if

$$
|f(\cos (\nu \pi / n))| \leqq 1, \quad \nu=0,1,2, \cdots, n,
$$

then for $p=1,2,3, \cdots, n$

$$
\left|f^{(p)}(x+i y)\right| \leqq\left|T_{n}^{(p)}(1+i y)\right| ; \quad-1 \leqq x \leqq 1,-\infty<y<\infty .
$$

The equality occurs only if $f(z) \equiv \pm T_{n}(z)$. 
Proof. Suppose $f(z)$ is not identically $\pm T_{n}(z)$. Then by Lemma IV there is a constant $c, c>1$, such that $\left|c f^{\prime}(x)\right| \leqq\left|T_{n}^{\prime}(x)\right|$ at the zeros of $T_{n}(x)$. Applying Theorem I to $c f(x)$ we find

$$
\left|f^{(p)}(x+i y)\right| \leqq \frac{1}{c}\left|T_{n}^{(p)}(1+i y)\right| .
$$

The case $f(x) \equiv \pm T_{n}(x)$ is also easily discussed.

In the above theorem in which an estimate of $f^{(p)}(z)$ was obtained throughout a strip of the complex plane the restriction that $f(z)$ have real coefficients was essential. However for points on the real axis the same bound for $\left|f^{(p)}(z)\right|$ holds even if $f(z)$ is allowed to have complex coefficients.

TheOREM III. Let $f(z)$ be a polynomial of degree $n$ satisfying $|f(\cos (\nu \pi / n))|$ $\leqq 1 ; \nu=0,1,2, \cdots, n$. Then for $p=1,2,3, \cdots, n$,

$$
\left|f^{(p)}(x)\right| \leqq \frac{n^{2}\left(n^{2}-1\right)\left(n^{2}-2^{2}\right) \cdots\left(n^{2}-(p-1)^{2}\right)}{1 \cdot 3 \cdot 5 \cdots(2 p-1)} .
$$

The equality occurs only if $f(z) \equiv \gamma T_{n}(z),|\gamma|=1$.

Proof. If $f(z) \not \equiv \gamma T_{n}(z)$ then by Lemma IV there is a constant $c, c>1$, such that $\left|c f^{\prime}(z)\right| \leqq\left|T_{n}^{\prime}(x)\right|$ at the zeros of $T_{n}(x)$. From the Remark to Theorem I it follows that

$$
\left|c f^{(p)}(x)\right| \leqq\left|T_{n}^{(p)}(1)\right|, \quad-1 \leqq x \leqq 1,
$$

for $p=1,2,3, \cdots, n$. To complete the proof of Theorem III we need only calculate $T_{n}^{(p)}(1)$.

Differentiating (11) $p$ times we obtain

$$
\left(1-z^{2}\right) T_{n}^{(p+2)}(z)-(2 p+1) z T_{n}^{(p+1)}(z)+\left(n^{2}-p^{2}\right) T_{n}^{(p)}(z)=0
$$

from which

$$
(2 p+1) T_{n}^{(p+1)}(1)=\left(n^{2}-p^{2}\right) T_{n}^{(p)}(1)
$$

follows. Now $T_{n}(1)=1$ so, using induction, we find that $T_{n}^{(p)}(1)$ is equal to the right-hand side of (19).

In Theorem III we have proved that the conclusions of Markoffs' theorem are true under the lighter hypothesis that $f(x)$ is bounded by 1 only at the $n+1$ points $x=\cos (\nu \pi / n) ; \nu=0,1,2, \cdots, n$. This raises the question if there are $n+1$ other points in the interval $(-1,1)$ with the same property. The answer is in the negative, however, for if $E$ is any closed set of points in $(-1,1)$ which does not include all the points $x=\cos (\nu \pi / n)$ then there is a polynomial of degree $n$ which is bounded by 1 in $E$, whose derivatives do not satisfy (19). To show this suppose that $E$ does not include the point $x=\cos (\lambda \pi / n)$. If 
$\lambda=0$ or $n$ the set $E$ is contained in an interval of length less than 2 , so an example is easily constructed. Suppose $\lambda \neq 0, n$ and let

$$
P(x)=\frac{\left(x^{2}-1\right) T_{n}^{\prime}(x)}{x-\cos \lambda \pi / n}, \quad f(x)=T_{n}(x)+\alpha P(x), \quad \alpha>0 .
$$

If $\epsilon$ is a small positive number the set $E$ is contained in the two intervals $(-1, \cos \lambda \pi / n-\epsilon)$ and $(\cos \lambda \pi / n+\epsilon, 1)$. Call this the set $E^{\prime}$. It is clear that if $\alpha$ is sufficiently small we shall have $|f(x)| \leqq 1$ in a neighborhood of the end points of the two intervals of $E^{\prime}$. Then the maximum of $|f(x)|$ for $x \in E^{\prime}$ (and this maximum is greater than 1) must occur in a small neighborhood of some point $\cos k \pi / n$ where $k \neq 0, \lambda, n$. At this maximum, say $x_{1}$, we have

$$
f^{\prime}\left(x_{1}\right)=T_{n}^{\prime}\left(x_{1}\right)+\alpha P^{\prime}\left(x_{1}\right)=0 .
$$

But

$$
\left|P^{\prime}(x)\right| \leqq A_{1}, \quad-1 \leqq x \leqq 1,
$$

and in a neighborhood of $\cos (k \pi / n)$

$$
\left|T_{n}^{\prime}(x)\right| \geqq A_{2}|x-\cos (k \pi / n)| \text {. }
$$

Then

$$
A_{2}\left|x_{1}-\cos (k \pi / n)\right| \leqq\left|T_{n}^{\prime}\left(x_{1}\right)\right|=\alpha\left|P^{\prime}\left(x_{1}\right)\right| \leqq \alpha A_{1} .
$$

Thus the maximum of $f(x)$ occurs within a distance $\alpha A_{1} / A_{2}=A_{3} \alpha$ of some point $\cos (k \pi / n)$ where $k \neq 0, \lambda, n$ and $A_{3}>0$ is independent of $\alpha$. Hence we have by the mean value theorem

$$
\begin{aligned}
T_{n}\left(x_{1}\right) & =T_{n}(\cos (k \pi / n))+\frac{1}{2}\left(x_{1}-\cos (k \pi / n)\right)^{2} T_{n}^{\prime \prime}\left(x_{2}\right), \\
P\left(x_{1}\right) & =\left(x_{1}-\cos (k \pi / n)\right) P^{\prime}\left(x_{3}\right)
\end{aligned}
$$

where $\left|x_{2}-x_{1}\right|<\left|x_{1}-\cos (k \pi / n)\right|, \quad\left|x_{3}-x_{1}\right|<\left|x_{1}-\cos (k \pi / n)\right|$. Then if

we have

$$
\left|T_{n}^{\prime \prime}(x)\right| \leqq A_{4}, \quad-1 \leqq x \leqq 1
$$

$$
\left|f\left(x_{1}\right)\right| \leqq 1+\frac{1}{2} A_{4} A_{3}^{2} \alpha^{2}+A_{1} A_{3} \alpha^{2}=1+A_{5} \alpha^{2} .
$$

Thus for all small $\alpha$ the polynomial

$$
F(x)=f(x)\left(1-\alpha^{3 / 2}\right)
$$

satisfies

$$
\begin{array}{rlr}
|F(x)| & <\left(1+A_{5} \alpha^{2}\right)\left(1-\alpha^{3 / 2}\right)<1, & x \in E^{\prime}, \\
\left|F^{(p)}(1)\right| & =\left(T_{n}^{(p)}(1)+\alpha P^{(p)}(1)\right)\left(1-\alpha^{3 / 2}\right)>T_{n}^{(p)}(1), \quad p=1,2,3, \cdots, n .
\end{array}
$$




\section{Application to entire functions}

Professor Szegö has pointed out to us that by a limiting process the previous results may be applied to entire functions.

THEOREM IV. Let $f(z)$ be an entire function, real for real $z$, which satisfies

(a) $|f(z)|=o\left(|z|^{1 / 2} \exp \pi|z|^{1 / 2}\right)$ uniformly as $|z| \rightarrow \infty$,

(b) $\left|f\left(n^{2}\right)\right| \leqq 1, n=0,1,2,3, \cdots$.

Then for $p=1,2,3, \cdots$ and $x \geqq 0$

$$
\left|f^{(p)}(x+i y)\right| \leqq\left|\frac{d^{p}}{d y^{p}} \cos \pi(i y)^{1 / 2}\right| .
$$

Proof. It is evident that $f\left(z^{2}\right)$ is an entire function of exponential type and is $o\left(|z| e^{\pi|z|}\right)$ as $|z| \rightarrow \infty$. According to a result of Valiron we may write $\left(^{5}\right)$

$$
f\left(z^{2}\right)=z \pi^{-1} \sin \pi z\left\{\frac{f(0)}{z^{2}}+\sum_{-\infty}^{\infty} \frac{(-1)^{\nu} f\left(\nu^{2}\right)}{\nu(z-\nu)}\right\} .
$$

Then

$$
f(z)=(z)^{1 / 2} \pi^{-1} \sin \pi(z)^{1 / 2}\left\{\frac{f(0)}{z}+2 \sum_{\nu=1}^{\infty} \frac{(-1)^{\nu} f\left(\nu^{2}\right)}{z-\nu^{2}}\right\} .
$$

Let

$$
\phi_{n}(z)=z(z-2) T_{n}^{\prime}(1-z)=c \prod_{\nu=0}^{n}\left(z-\lambda_{\nu, n}\right)
$$

where $\lambda_{\nu+1, n}-\lambda_{\nu, n}>0$ and $c \neq 0$. By Theorem II, if $-1 \leqq \delta \leqq 1$ and $0 \leqq x \leqq 2$,

$$
\left|\frac{d^{p}}{d z^{p}} \sum_{\nu=0}^{k} \frac{\delta_{\nu} \phi_{n}(z)}{\phi_{n}^{\prime}\left(\lambda_{\nu, n}\right)\left(z-\lambda_{\nu, n}\right)}\right| \leqq\left|\frac{d^{p}}{d y^{p}} T_{n}(1-i y)\right| .
$$

Here $k$ is any integer less than or equal to $n$. By a change of variable we obtain, if $0 \leqq x \leqq 4 n^{2} \pi^{-2},-\infty<y<\infty$,

$$
\left|\frac{d^{p}}{d z^{p}} \sum_{\nu=0}^{k} \frac{\delta_{\nu} \phi_{n}\left(\frac{1}{2} \pi^{2} n^{-2} z\right)}{\phi^{\prime}\left(\lambda_{\nu, n}\right)\left(\frac{1}{2} \pi^{2} n^{-2} z-\lambda_{\nu, n}\right)}\right| \leqq\left|\frac{d^{p}}{d y^{p}} T_{n}\left(1-\frac{1}{2} i \pi^{2} n^{-2} y\right)\right|
$$

Keeping $k, z$, and $\nu$ fixed we allow $n$ to increase and obtain

$$
\begin{gathered}
\lim _{n \rightarrow \infty} T_{n}\left(1-\frac{1}{2} \pi^{2} n^{-2} z\right)=\cos \pi(z)^{1 / 2}, \\
\lim _{n \rightarrow \infty} \phi_{n}\left(\frac{1}{2} \pi^{2} n^{-2} z\right)=\pi(z)^{1 / 2} \sin \pi(z)^{1 / 2}, \quad \lim _{n \rightarrow \infty} 2 \pi^{-2} n^{2} \lambda_{\nu, n}=\nu^{2} .
\end{gathered}
$$

(5) G. Valiron, Sur la formule d'interpolation de Lagrange, Bulletin des Sciences Mathématiques, vol. 49 (1925), pp. 203-224. Compare G. P6lya and G. Szegö, loc. cit., vol. 1, p. 117, Problem 165. 
Also

$$
\frac{1}{2} \pi^{2} n^{-2} \phi_{n}^{\prime}\left(\lambda_{\nu, n}\right)= \begin{cases}\pi^{2} & \text { if } \quad \nu=0 \\ \frac{1}{2}(-1)^{\nu} \pi^{2} & \text { if } \quad 0<\nu<n .\end{cases}
$$

Thus we obtain

$$
\left|\frac{d^{p}}{d z^{p}} \frac{(z)^{1 / 2} \sin \pi(z)^{1 / 2}}{\pi}\left\{\frac{\delta_{0}}{z}+2 \sum_{\nu=1}^{k} \frac{\delta_{\nu}(-1)^{\nu}}{z-\nu^{2}}\right\}\right| \leqq\left|\frac{d^{p}}{d y^{p}} \cos \pi(i y)^{1 / 2}\right|
$$

for all $\delta_{\nu}$ which satisfy $-1 \leqq \delta_{\nu} \leqq 1$. Now $k$ is an arbitrary positive integer, so the theorem follows.

We might say that Theorem IV is imperfect because in the passage to the limit from polynomials to entire functions we have lost touch with the conditions of equality. These could be discussed by reformulating Theorem I to make it apply to entire functions. A similar limiting process yields an analogue of Theorem III.

UNIVERSITY OF ILLINOIS,

URBANA, ILL.

StANFORD UNIVERSITY,

Stanford University, Calif. 